\title{
Dinamiche degli affetti nel romanzo italiano del Seicento
}

\section{Dynamics of "Affetti” in Seventeenth-century Italian Novel}

\author{
Simona Morando \\ Università degli Studi di Genova \\ simona.morando@unige.it
}

\begin{abstract}
The fortune of seventeenth-century Italian romance among readers is also based on an effective representation of passions. In this essay some examples from novels are offered to enlighten the major affective dynamics: love, in the case of La Stratonica by Luca Assarino (1635), and heroism, in the case of Demetrio moscovita by Maiolino Bisaccioni (1639), noting how the first novel imitate and exalt the traditional elegiacs modalities, while the latter sink in the core of the seventeenth-century most urgent matters, such as the Reason of State. Treatises on the passions ("affetti") of that time, mentioned here for the exemplary case of Tommaso Buoni, supports the switch from classical categories towards a modern vision of human affections.
\end{abstract}

Keywords: affetti, passions, novel, XVIIth century, Reason of State

Tra i molti tentativi di riabilitazione del romanzo secentesco italiano, che purtroppo non ha mai goduto di una buona considerazione critica e che ancora adesso viene avvertito come un fenomeno minore rispetto allo sviluppo di altri generi letterari coevi italiani, per non parlare dello sconfortante raffronto con la storia europea del romanzo barocco, c'è anche quello di vedervi l'origine della moderna questione delle passioni. Si vorrebbe cioè ritrovare nel romanzo del Seicento l'inizio e il profilarsi della moderna coscienza individuale, il nuovo «mito» della modernità, secondo quanto ci ha utilmente insegnato Watt (1996), l'espandersi della sensibile interiorità. Chi ha studiato maggiormente questa linea è Roberta Colombi che, nell'introduzione al suo libro Lo sguardo che «s'interna». Personaggi e immaginario interiore nel romanzo italiano del Seicento, vede nella narrativa barocca una nuova immagine 
dell'uomo che, dice, «sembra possa rappresentare l'inizio di una modernità intesa come coscienza della complessità, che in questi romanzi del Seicento si esprime attraverso la delineazione di un personaggio maggiormente caratterizzato» (Colombi, 2002, p. 21). Rispetto alla bidimensionalità dei paladini dell'epica cavalleresca, rispetto anche alla funzionalità altamente emotiva dei personaggi tassiani (che insegnano comunque moltissimo alla rappresentazione degli affetti secentesca), i personaggi del romanzo risalterebbero come figure a tutto tondo, con sfumature e profondità.

Ora, questa linea interpretativa ha delle ragioni. In queste poche pagine si tenterà di vagliarne il vero e ricalibrare la questione proponendo di cogliere la modernità delle passioni non tanto in relazione all'invenzione della coscienza - che forse non entra mai veramente in gioco nella letteratura di consumo del tempo - ma in relazione al dialogo tra singolo e società, singolo e storia. Per anticipare il punto di vista di chi scrive, si ritiene che la profondità dei personaggi romanzeschi secenteschi non sia messa a punto particolarmente in presenza della rappresentazione del sentimento amoroso (probabilmente la favola pastorale, con i suoi pastori e le sue ninfe in maschera, su fondali di cartone, osa maggiormente nella analisi delle reticenze, delle titubanze, degli eccessi e degli ardori dell'amore), ma in presenza di quei sentimenti vari e difficili che appartengono agli uomini e ai personaggi di potere, quei sentimenti che decifrano il rapporto, appunto, tra l'uomo singolo e la Storia. È in questa casistica, soprattutto, che i romanzieri affinano gli strumenti della rappresentazione del personaggio. L'eros e tutta la sfera della passione amorosa, infatti, restano sostanzialmente temi da esibire, anche con sfarzo, ma per censurarne la pericolosa interiorità. I sentimenti invece che, figli dell'epica storica, affinano il vero grande tema barocco, ossia il rapporto singolo-Stato, dentro i gangli della Storia, sono esercitati con dovizia di prospettiva.

Oltre a queste due tematiche, che si inscrivono bene nella tradizione affettiva letteraria, va però segnalato il caso del Calloandro fedele di Giovan Ambrosio Marini, romanzo cult e di successo per tutta la seconda metà del Seicento e ristampato fino al secolo successivo, che individua una nuova realtà interiore del personaggio: il disorientamento, che non rientra nelle categorie tomistico-aristoteliche delle passioni, le quali ancora incidono sulla teoria secentesca, se non per una vicinanza con l'amor di sé o il mancato amor di sé. «Dimmi ti prego, Foriano, chi egli è, se vuoi ch'io sappia chi son io. Ho perduto il conoscimento di me stesso, e perderò anco la vita se non mi scuopri la verità» (Romanzieri del Seicento, 1974, p. 270), esclama il Cavalier di Cupido con i suoi baffi posticci dentro il suo labirinto. Ma le strategie dell'introspezione non sono ancora affinate, neppure in questo caso.

Procediamo con qualche esemplificazione. Storicamente si data la nascita del romanzo moderno italiano, come è noto, con Eromena di Francesco Biondi, 1624, uscito appena un anno dopo l'opera che conclude idealmente la parabola gloriosa del poema in ottave, cioè Adone di Giovan Battista Marino. Però questo romanzo inaugura a sua volta il filone avventuroso e cavalleresco del romanzo, tutto dedito 
all'azione e alla peripezia, in cui gli affetti forti, amorosi, filiali, familiari, sono come parti dell'ingranaggio della peripezia medesima. Una specifica attenzione all'analisi degli affetti è invece prioritaria nel secondo romanzo che tradizionalmente si nomina nella cronologia narrativa barocca, cioè L'Amorosa Clarice di Ferdinando Donno, pubblicato a Venezia nel 1625 (ma lo scrive prima dell'Eromena del Biondi in verità, nel 1619), che mette al centro la vicenda di Clarice, una donna che nella sua Manduria, si innamora del giovane Lelio, senza esserne ricambiata, fino a che quest'ultimo non trova la morte a Parigi, suggerendo anche alla protagonista un gesto estremo che però verrà evitato da una riappacificante apparizione in sogno di Lelio.

In questa breve trama si tradisce la consapevolezza di una tradizione romanzesca molto forte: il modello è infatti l'Elegia di Madonna Fiammetta di Boccaccio, fortunatissima nel secolo XVI per edizioni e rifacimenti, abecedario vero dell'analisi amorosa, della fisiologia d'amore. Nonché modello per la narrazione in prima persona, di stampo elegiaco, cosa che puntualmente anche Donno imita. Il romanzo non ha grande successo, causa proprio la volontà insistita di rifarsi a Boccaccio, ma merita di essere annoverato come uno dei primi tentativi di romanzo moderno che mette a fuoco il tema, anch'esso «elegiaco», delle passioni. Come dirà il Manzini col Cretideo (1637) il romanzo è una «gloriosa machina» fabbricata dall'ingegno e come tale non ama indugiare sull'analisi delle intimità. Il che è vero e non vero, come si vedrà con l'uscita, nel 1635, della Stratonica di Luca Assarino. Probabilmente però Clarice non ha molto da aggiungere alla già fortunata ricezione di Fiammetta e la strategia del racconto in prima persona e in punta di lacrime non è, a quel punto, una novità. Così come le variazioni introdotte da Donno (il travestimento in abiti maschili della protagonista, la morte dell'amato Lelio, carattere buono fino alla fine, lo stato nubile di Clarice) tolgono sale alla vicenda boccacciana, di infedeltà e obliqui sentimenti. Si tratta di un altro caso in cui ci si impegna a censurare nei fatti Boccaccio, come in molti punti tra Cinque e Seicento. Ma probabilmente il mancato successo sta maggiormente nello stile che non ha ancora trovato una sua scorrevolezza diegetica, ma si impenna in interrogazioni retoriche, in aggettivazioni ripetute, in sintassi liriche, in virtuosismi come in questo stralcio che mira proprio a individuare la rappresentazione della passione della protagonista:

E qui io assalita nel mesto petto da repentino e orrido gelo, con pie' molesto da vena in vena si conferiva, con tosti passi da membro in membro si diffondeva e me rendendo tutta tremante da capo a' piedi, risomigliava in lubrica riva tremolo pioppo sfracolato nell'altezza dell' aria da forzevoli venti. (Donno, 1979, p. 232)

Non c'è competizione con l'affabilità del Biondi anche sullo stesso piano affettivo:

Solcava l'onde il principe Matteone, con vari affetti di contento e di dolore: lacerandolo la memoria delle sue colpe e consolandolo la speranza di trovare il fratello, per ammendare con effetti contrari i difetti passati. (Romanzieri del Seicento, 1974, p. 94) 
Non è ozioso il paragone: serve a stabilire che il successo del romanzo passa anche attraverso la semplificazione delle strutture linguistiche e retoriche, che evidentemente l'adozione della sintassi e dell'analisi affettiva boccacciane non ha consentito. Certamente l'amore è il tema che il libro di Donno pone al centro specifico del romanzesco, motore primo della narrazione. E di fatto anche Colombi (2002, p. 9) afferma che «la novità del romanzo secentesco infatti, riconosciuta persino dai contemporanei, è quella di porre al centro l'amore come principale argomento».

Prendiamo il caso del romanzo che più di ogni altro è stato additato come esemplare dell'analisi della passione amorosa. Si tratta del vero best-seller del secolo, La Stratonica del genovese Luca Assarino pubblicato prima a Viterbo nel 1634 e poi a Venezia e in altre città nel 1635, infine completato con un terzo libro nel 1637 (oggi Assarino, 2003). Subito tradotto in molti paesi europei, e sempre ristampato in Italia, primo tra i romanzi letti nel secolo col Calloandro di già citato, La Stratonica si caratterizza per il forte tratto analitico degli affetti, approfondendo i modelli del d'Urfè, del Camus e le effusività di Tasso, già amate da un altro ligure, Carlo della Lengueglia, autore di molti romanzi erotico-galanti, e da lui estenuate in una cornice ingegnosa e aristocratica. Assarino molto astutamente mutua questa analisi affettiva dalle caratterizzazioni psicologiche che in Italia si era ben evidenziate nel romanzo secentesco di tema religioso, come il Sant'Eustachio di Giovan Battista Manzini, pubblicato nel 1631, e che infatti proprio in suolo genovese trova una sua riuscita variante nel romanzo religioso di Anton Giulio Brignole Sale (cui peraltro è dedicata la stampa viterbese della Stratonica), cioè la fortunata Maria Maddalena peccatrice e convertita del 1636 (Genova: Ferroni) che senz'altro centra l'immaginario secentesco con la figura voluttuosa, preziosa, ambigua e poi spiritualmente mistica della donna convertita da Gesù.

Nel libro di Assarino l'avventura cede il passo all'anatomia dei sentimenti. La storia dell'amore di Antioco, per la sua giovane matrigna Stratonica, moglie di Seleuco (che di lei si innamora guardando un suo ritratto, secondo il noto topos letterario) e figlia del mitico Demetrio (a cui Assarino dedica un altro romanzo nel 1643), era già nota per via di Plutarco, Petrarca, Bandello ; ma Assarino trasforma la principessa da oggetto d'amore a soggetto innamorato a sua volta perché nutre una passione smodata per Antioco, complicando così la trama sentimentale. I personaggi di Assarino, come nota la Colombi, sono immersi nel loro silenzio e pensano a dismisura, rendendoci lettori dei loro pensieri e non spettatori delle loro azioni, quasi azzerate. Si tratta di un approfondimento affettivo al quale non darei i connotati di una introspezione di coscienza, ma di una estensione degli strumenti elegiaci. Non è però possibile credere che Assarino sia un campione della scoperta dell'io moderno: la forza del suo romanzo sta nella sua agilità di stile e di trama, nel suo essere concentrato intorno ad un caso erotico che viene indagato in dettaglio e nel saper collocare questo stesso caso (soprattutto col terzo libro del 1637) all'interno di una corte descritta come un luogo orribile. 
La storia è narrata da una voce partecipe che interloquisce retoricamente, come Tasso, con i suoi personaggi, amplificando il pathos («Non moristi, Seleuco, di dolcezza, perch'era impossibile il morir in bocca alla tua vita. Sentì ben l'anima tua epilogati in quel bacio tutti que' gusti che amor può dare»: Assarino, 2003, p. 27). Il pathos viene applicato alla lucida disamina degli innamoramenti: quello di Seleuco folgorante e immediato, e quello di Antioco per Stratonica e purtroppo destinato a diventare malattia infermante che si esercita in questo tipo di analisi elegiaca, corroborata da interrogazioni retoriche «Queste quai vie sono? Ove si corre Antioco? Tu machini tradimenti? tradimenti tanto più atroci, quanto, che sono contra l'onore? Il tuo cuore può haver recesso ove s'asconda un pensier così sozzo com'è adulterar contro il padre? E vivi? E respiri? Ed hai fronte per sostener l'altrui guardo? Misero, ed in che cosa fondi le tue speranze, quando anco fussero speranze giuste [...]» (Assarino, 2003, p. 38).

La compattezza dei primi due libri si perde nel terzo, tra omaggi a Genova e ai suoi poeti, diversioni novellistiche e differimento nella soluzione della trama, dovuta al medico di corte che ingegnosamente riesce a persuadere il re a privarsi della moglie per il bene del figlio. Il romanzo diviene così una storia edificante che fa leva soprattutto sull'amore paterno e sul mantenimento dell'onor dello stato, che alla fine è il punto importante e conclusivo della faccenda.

Ma proprio a quel punto tendono molte vicende del romanzo secentesco, anche quando partono dalla esibizione di sentimenti diversi, come l'amore, appunto.

Bisogna infatti considerare come uno dei modelli importanti del romanzo italiano sia l'Argenis di John Barclay del 1621 prontamente tradotto dal latino da Francesco Pona (altro iniziatore del romanzo, se si considera lo stravagante caso tra novella e romanzo della sua Lucerna) con L'Argenide di Giovanni Barclaio tradotta da Francesco Pona, pubblicata a Venezia nel 1629. Qui il personaggio inquieto di Poliarco occupa la scena con i suoi sentimenti di onore e di amore per la bella Argenis, sullo sfondo delle guerre siciliane di un tempo imprecisato continuamente alludente però alle guerre di religione contemporanee all'autore. Nelle figure dei protagonisti maschili Barclay rappresenta tre ipotesi di governo, di cui solo quello di Poliarco è giusto e buono, dettato da onestà e temperanza. In un passaggio iniziale, infatti, vediamo come i sentimenti del giovane siano ben dettagliati proprio in reazione al suo orgoglio ferito di principe: «Ciò udito Arcombroto e Timoclea, unitamente si pongono à rimirare Poliarco. Egli, pallido, e tremante non già per essere consapevole à se medesimo di delitto, mà per lo sdegno d'esser posto in pericolo, non sopportando la Virtù d'essere oltraggiata, tornava à chiedere al servo, se le cose che rifferiva eran vere $[\ldots . . . »$ (Barclay e Pona, 1629, p. 22). Pallore e tremore possono essere nella rappresentazione della dinamica delle passioni segni di molti affetti: di amore, innanzitutto, e come qui, di sdegno. Lo sdegno, o indignazione, però è un affetto che i trattatisti del tempo (facciamo qui il caso di Buoni 1605) attribuiscono come proprio dell'uomo che vive in società, come donato da Dio allo scopo di avvertire la 
violazione della giustizia e ripristinarla. Buoni (1605, p. 134v) fornisce questa definizione:

illustre affetto, per il quale si concitasse ad honoreuole odio per estirpare simile grave impedimento alla vita sociabile, e così si conservasse l'ordine di giustitia inviolabilmente, il quale affetto è detto indignatione dai latini, per contenere in un certo sdegno conceputo per cose, che parreno haver dell' indegno; il che si può dir che sia una certa tristezza accompagnata da un certo generoso odio, nato da beni gravi, che si vedono goder da coloro che non sono di quelli meritevoli; e conseguentemente godendosi da coloro, che sono meritevoli s'allegra; è tristezza, perché ci affligge il cuore, et anco si manifesta talhora esteriormente nel corpo; et perciò al dolore si può riferire accompagnata da un certo generoso odio.

Ira, odio e sdegno, quando sono frutti di una mancanza di giustizia, che viene avvertita come brutale e crudele, sono dunque sentimenti necessari per i principi che si apprestano a diventare dei buoni re.

Con l'Argenis si data l'inizio di un filone storico-eroico, intrecciato ai valori dell'amore, che verrà ben recepito dagli autori italiani. In primis dal principe dell'Accademia degli Incogniti, Francesco Loredano, che nel 1635 pubblica La Dianea, romanzo a chiave in cui il personaggio storico meglio evidenziato è il «mitico» Wallenstein. Ma andrei al romanzo più importante del filone. L'autore è Maiolino Bisaccioni, agente politico e uomo d'arme dalle molteplici avventure. La sua opera, che di fatto introduce il romanzo storico, è il Demetrio moscovita, pubblicato prima nel 1639, poi nel 1643 e di nuovo nel 1649. Bisaccioni, che aveva trattato storiograficamente le guerre nel Commentario delle guerre successe in Alemagna dal tempo che il re Gustavo di Svetia si levò di Norimberga (Venezia 1633-1642) e che teneva fede alla storia come «ammaestratrice», secondo il detto di Agostino Mascardi e della sua Arte historica del 1636, offre qui una prova originale, in linea con le modalità di riscrittura «tragica» della storia. Non ci addentriamo qui nello spinoso ma necessario dibattito tra storia e romanzo, intavolato lungamente dagli scrittori secenteschi e relativo allo stile, pro e contro il laconismo. Le strade del romanzo e della storiografia sembrano, alla fine di questo dibattito, correre parallele, ma restava agli autori l'onere di sciogliere la questione.

Proprio per questo è coraggiosa e significativa la soluzione del Bisaccioni, sentenzioso e tacitiano e decisamente a favore della storia narrata che si fa romanzo. La sua produzione è soprattutto novellistica ed è anche fine traduttore dal francese (dalla de Scudéry ad esempio). Il Demetrio è il suo unico romanzo, oggi disponibile anche in edizione moderna (Bisaccioni, 1992): un «quasi-romanzo», secondo il moderno curatore Taddeo, che racconta l'ascesa e la caduta del falso Demetrio nella Russia di primo Seicento, basandosi, come detta il suo rigoroso metodo compositivo, su fonti storiche e testimonianze di prima mano e quindi non occhieggiando al favoloso come nelle novelle. Di romanzesco qui, come ha visto bene Taddeo, c'è la 
configurazione dei personaggi, divisi tra eroi e antieroi, c'è l'analisi appunto dei moti dell'animo. Non dobbiamo aspettarci aperture straordinarie in questo senso perché il romanzesco è una cifra che arriva nel testo a onde tardive: lo scrupolo storiografico impone all'autore un incipit geografico e storico con stile asciutto, ma qualche intervento soggettivo ( $«$ Misero Demetrio! Di cui mi accinsi a scrivere, ch'anco di te mi dimenticai», Bisaccioni, 1992, p. 15), poi si alternano i registri, a tratti sentenze storiche («Dure invero sono le massime del regno, e però si dipinge il Prencipe armato per dimostrarlo impenetrabile dalle cose di fuori...», Bisaccioni, 1992, p. 31) e sempre in prima persona, da storico-narratore omnisciente, a larghi tratti soggettive animate quasi tassiane: «Demetrio $[\ldots]$ non fuggì no, ma come toro feroce, sempre col fronte all'inimico, retrocedendo il passo, fu coperto dalla notte, che d'una folta caligine li fece trincera» (Bisaccioni, 1992, p. 41). Alle quali soggettive si aggiungono, frutto di finzione, lettere d'amore tra Demetrio e Anna Marina, altre epistole, discorsi diretti, citazioni poetiche. La narrazione in prima persona è di fatto importante per trasmettere la pietà e il senso di tragedia con cui la voce narrante guarda al suo Demetrio, eroe buono e sventurato, ed è importante per formulare un giudizio storico partecipe.

La novità nell'analisi delle passioni nel Demetrio risiede però in questo: che la voce narrante mentre rivela le dinamiche affettive di Demetrio intende segnalare proprio al lettore un corretto esercizio educativo sugli affetti. Prendo la pagina in cui Demetrio e la sua amata vengono descritti a colloquio, liberi di manifestare reciprocamente il loro amore. Bisaccioni (1992, p. 24) commenta:

Chi disse l'amore figlio dell'ozio, non amò già mai, se non sozzamente, e chi lo stimò voglioso di tutto il cuore, e tiranno d'una mente, o non fu cavaliero, o non amò da cavaliero; egli non sa viver solo in nobil cuore, anzi solo morirebbe di rabbia, o tralignarebbe in quel mal nato, chè la corrutela de' sensi ; vuole per compagno l'onore, col quale tratta così nobilmente, che quegl'istesso, ch' alberga l'uno e l'altro nel petto, non può discernere a chi debba la maggioranza dell'affetto, poiché se ama, si muove a fine d'azioni onorate, e se maneggia cose d'onore, par che le tratti per dimostrarsi degno amante.

Nella disamina delle passioni Bisaccioni non fa che riannodare i fili di una fitta tradizione che affonda nel codice cavalleresco medievale, nella fisiologia degli affetti che vi vengono catalogate, per sugellare una continuità umana senza tempo. Per questa continuità, in virtù d'essa, amore e onore si legano visceralmente e anche il moderno Demetrio asseconda e consuma dentro di sé un protocollo noto. Ma questo antico codice si incastra come un diamante in un animo, quello di Demetrio, che viene chiuso da Bisaccioni in un silenzio severo e concentrato sulla sua missione principesca. «Uno dei più chiari segni di nascita grande è il parlar poco, maestoso, e con franchezza; condizioni, che né meno le apprende tutte, benché vi studii, il volgo» (Bisaccioni, 1992, p. 27). Demetrio parla poco con se stesso e pochissimo con gli altri, al contrario dei personaggi di Assarino che continuamente dialogano 
con la propria anima d'amore. Demetrio agisce, al contrario dei protagonisti della Stratonica, e, per effondere i suoi sentimenti, scrive lettere, anch'esse trattenute ma trapelanti il calore dell'animo nei fatti della storia. L'educazione sentimentale di questo romanzo passa attraverso i silenzi, attraverso le sentenze pronunciate dalla voce narrante e dai fatti, veri esempi di giustizia. Il momento dell'assassinio di Demetrio, compiuto dalla folla inferocita sobillata dai traditori, non cambia le cose. Il dettagliato resoconto del tentativo di fuga di Demetrio che si getta dalla finestra della sua camera, procurandosi moltissime ferite, e poi il soccorso che lo riporta in vita solo perché si compia il vero massacro sono i fatti che sostituiscono le analisi delle passioni, delle vittime come dei carnefici. Ma nel corpo di Demetrio le fibre ancora palpitanti nell'agonia riportano passioni più incisive di quelle che avrebbero potuto essere descritte a parole e la sua anima, che ha tante porte quante ferite per uscire dal corpo, è anch'essa un fatto, cosa concreta del vero personaggio Demetrio:

Accorsero in quella camera alcuni principali e parlarono all'infelice moribondo di cose, che non si poterono risapere, poiché per farle più occulte, fu di subito il Firstemburgo ammazzato, indi rivoltate l' armi fumanti del sangue todesco in Demetrio, fu egli da cento e cento colpi ferito, e aperte più vie di fuggire a quell'anima stanca sotto il peso della fortuna, e tante furono le ferite, che a pena ebbero luogo i spiriti vitali di dar gli ultimi tremori; quindi il cadavere, le cui fibre palpaitavano ancora, fu lasciato in preda al popolaccio, e uno della più vile canaglia, trovata una fune il cinse là dove si congiungono le cosce al corpo, e chiamati molti altri in aiuto, si pose a trascinarlo per le strade tra il loto e le più sordide immondicie. (Bisaccioni, 1992, p. 273)

La strategia analitica di Bisaccioni è dunque perfetta, asciutta, laconica e incisiva nel dare conto delle passioni attraverso il silenzio. L'eroismo di Demetrio deve riverberarsi nel dolore e direi nell'orrore che il lettore dovrebbe a questo punto provare alla lettura della pagina conclusiva del primo libro del romanzo. L'orrore non è un sentimento secondario, data l'esibizione del corpo martoriato del sovrano, che poi, in un passo che non ho riportato in esteso, verrà esibito alla madre anziana, la quale solleciterà appunto la pietà. Terrore e pietà del resto, erano già state annunciate nel titolo del romanzo, in quella «istoria tragica» che deve sintonizzare il lettore proprio sui fulcri passionali della tragedia secondo Aristotele, terrore e pietà, appunto. Non mi pare un caso che anche il trattato sugli affetti che si è qui citato, Buoni 1605, nel suo capitolo undicesimo dedicato ai dolori umani, imposti il discorso a partire dalla crudeltà dei tiranni, che causano sofferenze indicibili ai singoli e ai popoli, a fronte della quale si pone la morte del giusto principe, fonte di dolore inesauribile per la corte, per la regina, e di cordoglio per tutta la nazione. Il capitolo dedicato al dolore è dunque altamente politico e legato al sentimento del potere e dello stato. Facendo appello a questo sentimento e anche alle radici più vivaci della tragedia greca, sempre riproposta e riscritta in senso politico anche nel Seicento, Bisaccioni fa della morte di Demetrio un atto di catarsi per il lettore, in preda al 
terrore e alla pietà. Questa catarsi, questa purgazione delle passioni, deve portare per lui ad una maggiore consapevolezza, da parte sempre dell'empatico lettore, della verità storica e delle dinamiche del potere. Un'ambizione didattica dichiarata, dunque, che mescola lucrezianamente il dolce all'amara medicina, dove il dolce può essere visto non certo nella disgraziata vicenda di Demetrio, ma nell'efficace stile laconico e breve dell'autore. 


\section{BIBLIOGRAFIA}

Assarino, L. (2003). La Stratonica (R. Colombi, Ed.). Lecce: Pensa.

Barclay, J., Pona, F. (1629). L'Argenide di Giovanni Barclaio tradotta da Francesco Pona. Venezia: Giovanni Salis.

Bisaccioni, M. (1992). Il Demetrio Moscovita. Istoria tragica (E. Taddeo, Ed.). Firenze: Leo S. Olschki.

Buoni, T. (1605). Discorsi Academici delle grandezze del microcosmo. Parte seconda de' mondi [...]. Venezia: Colosini.

Capucci, M. (Ed.) (1974). Romanzieri del Seicento. Torino: Utet.

Colombi, R. (2002). Lo sguardo che «s'interna». Personaggi e immaginario interiore nel romanzo italiano del Seicento. Roma: Aracne.

Donno, F. (1979). Opere (G. Rizzo, Ed.). Lecce: Milella.

Watt, I. (1996). Myths of Modern Individualism: Faust, Don Quixote, Don Juan, Robinson Crusoe. Cambridge: Cambridge University Press.

Watt, I. (1998). Miti dell'individualismo moderno. Faust, don Chisciotte, don Giovanni, Robinson Crusoe (M. Baiocchi, M. Gnoli, Eds.). Roma: Donzelli (Edizione oryginale 1996). 